\title{
Acinetobacter baumannii nosocomial pneumonia: is the outcome more favorable in non-ventilated than ventilated patients?
}

\author{
Ya-Sung Yang ${ }^{1}$, Yi-Tzu Lee ${ }^{2,3}$, Tsai-Wang Huang ${ }^{4}$, Jun-Ren Sun ${ }^{5}$, Shu-Chen Kuo ${ }^{2,6}$, Chin-Hsuan Yang ${ }^{1}$, Te-Li Chen ${ }^{2 *}$,
} Jung-Chung Lin ${ }^{1}$, Chang-Phone Fung ${ }^{2}$ and Feng-Yee Chang ${ }^{7}$

\begin{abstract}
Background: Acinetobacter baumannii hospital-acquired pneumonia (HAP) is associated with a high mortality worldwide. Non-ventilated patients with HAP (NVHAP) caused by nosocomial pathogens are reported to have a more favorable outcome than those with ventilator-associated pneumonia (VAP). The current study was designed to determine whether bacteremic patients with A. baumannii NVHAP also have a lower mortality than those receiving assisted ventilation.

Methods: This retrospective 10-year study was conducted at a 2900-bed teaching hospital located in Northern Taiwan. The population consisted of 144 patients with A. baumannii bacteremia and HAP. Of these 96 had VAP and 48 had NVHAP. Charts were reviewed for demographic characteristics, comorbidities, clinical manifestations, antimicrobial susceptibility, and 14-day mortality. Clonal relationships were determined by molecular typing.

Results: There were no significant differences between the two groups in comorbidities (Charlson scores). Patients with NVHAP were more likely to have developed bacteremia earlier, outside the ICU and undergone fewer invasive procedures. They had significantly lower APACHE II scores, fewer bilateral pneumonias and lower rates of antimicrobial resistance. No specific clones were identified in either group. The unadjusted (crude) 14-day mortality rates were not significantly different between the groups (NVHAP 43.8\% vs. VAP 31.3\%, $p=0.196$ ). The adjusted 14-day mortality risk was significantly lower in ventilator-assisted patients (odds ratio $=0.201 ; 95 \%$ confidence interval $=0.075-0.538 ; p=0.001$ ).

Conclusions: Patients with bacteremic NVHAP and VAP caused by A. baumannii had similar crude mortality rates, but on logistic regression analysis those receiving ventilator assistance had a significantly lower mortality. This may have been due to better airway protection, more intensive monitoring with earlier diagnosis and treatment in patients with VAP, greater innate susceptibility to infection in those with NVHAP and differences in the virulence of A. baumannii.
\end{abstract}

Keywords: Acinectobacter baumannii, Pneumonia, Hospital acquired, Ventilator

\section{Background}

The three most clinically relevant, but phenotypically undifferentiated Acinetobacter species, Acinetobacter baumannii, Acinetobacter nosocomialis (formerly Acinetobacter genomic species 13TU), and Acinetobacter pittii (formerly Acinetobacter genomic species 3), have emerged as important nosocomial pathogens [1]. The prevalence of health care associated infections caused by Acinetobacter is

\footnotetext{
*Correspondence: tecklayyy@gmail.com

${ }^{2}$ Institute of Clinical Medicine, School of Medicine, National Yang-Ming University, Taipei, Taiwan

Full list of author information is available at the end of the article
}

increasing among patients in intensive care units (ICUs) and immunocompromised hosts [2-5]. The most common clinical condition associated with these microorganisms is hospital-acquired pneumonia (HAP), particularly for patients receiving mechanical ventilator assistance [6].

Among the three Acinetobacter species, A. baumannii is associated with a poorer outcome and higher rates of antimicrobial resistance [7]. Several A. baumannii virulence factors have been identified. These include CsuA/BABCDE, a chaperone-usher pili assembly system, the siderophoremediated iron acquisition system and outer membrane protein A (OmpA) [8,9]. The mortality rates for bacteremia

\section{Ciomed Central}

(c) 2013 Yang et al.; licensee BioMed Central Ltd. This is an Open Access article distributed under the terms of the Creative Commons Attribution License (http://creativecommons.org/licenses/by/2.0), which permits unrestricted use, distribution, and reproduction in any medium, provided the original work is properly cited. 
caused by $A$. baumannii range from 29.8 to $58.6 \%$ $[7,10,11]$. It has been previously reported that nonventilated patients with HAP (NVHAP) caused by nosocomial pathogens have a better outcome than those with ventilator-associated pneumonia (VAP) [12-14]. It is not known whether this applies to A. baumannii as well. The current retrospective study was designed to determine whether patients with $A$. baumannii bacteremic NVHAP have a better outcome than those with bacteremic VAP.

\section{Methods}

\section{Study population}

This study was conducted at Taipei Veterans General Hospital (T-VGH) during a ten-year period from January 2000 to December 2009. T-VGH is a 2900-bed tertiary-care teaching hospital located in Taipei, Taiwan. The data were analyzed at the Tri-Service General Hospital (TSGH), National Defense Medical Center in Taipei, Taiwan.

Charts were reviewed for all patients with at least one positive blood culture for $A$. baumannii who had symptoms and signs of infection. The inclusion criteria for A. baumannii pneumonia [15] consisted of: a) at least one positive respiratory sample (sputum, endotracheal aspirate, or broncho-alveolar lavage [BAL]) for A. baumannii obtained within 48 hours before or after the first positive blood culture; b) a clinical course compatible with pneumonia, including symptoms of acute respiratory infection and acute infiltrates on a chest radiograph; and c) the positive blood culture could not be attributed to another source of infection. NVHAP was defined as pneumonia that occurred 48 hours or more after admission in non-ventilated patients. VAP was defined according to the 2005 American Thoracic Society/Infectious Diseases Society of America (ATS/IDSA) guidelines [16] as pneumonia that occurred more than 48 hours after endotracheal intubation. The quantitative microbiologic criteria for the diagnosis of HAP required a bacterial count exceeding a threshold of $10^{3} \mathrm{cfu} /$ $\mathrm{mL}$ in a protected specimen brushing, $10^{4}$ or $10^{5} \mathrm{cfu} / \mathrm{mL}$ in a BAL fluid specimen or $10^{6} \mathrm{cfu} / \mathrm{mL}$ in an endotracheal aspirate [16]. Patients $<18$ years of age and those with incomplete medical records were excluded. All the clinical samples were taken as part of standard care. The standards of patient care did not significantly change during the study period. Mechanical ventilated patients not admitted to an ICU were treated in a respiratory care center, respiratory ward or common ward. The protocol was approved by the T-VGH and TSGH Institutional Review Board (approval number: 2011-10-012IC and 2-101-05-074) with a waiver for informed consent.

\section{Data Collection}

Medical records were reviewed to extract clinical information, including demographic characteristics, underlying diseases, Charlson comorbidity score [17], duration of stay in an ICU, hospital stay, administration of individual antimicrobials, the presence of a ventilator, central venous catheters, a nasogastric tube, or a foley catheter at the time of onset of bacteremia.

Chronic lung diseases other than chronic obstructive pulmonary disease (non-COPD chronic lung disease) included asthma, bronchiectasis, pulmonary fibrosis, and chronic pulmonary tuberculosis. Immunosuppressive therapy was defined as receipt of cytotoxic agents within 6 weeks, corticosteroids at a dosage equivalent to or higher than $10 \mathrm{mg}$ of prednisolone daily for more than 5 days within 4 weeks, or other immunosuppressive agents within 2 weeks prior to the onset of bacteremia. Neutropenia was defined as an absolute neutrophil count $<0.5 \times 10^{9}$ neutrophils/L. Recent surgery was defined as operations performed within 4 weeks prior to the onset of bacteremia. Chronic kidney disease was defined as an estimated glomerular filtration rate $<60$ $\mathrm{mL} / \mathrm{min} / 1.73 \mathrm{~m}^{2}$ for at least 3 months prior to admission. The severity of illness was evaluated using the acute physiology and chronic health evaluation II (APACHE II) score [18] within 24 hours prior to bacteremia onset.

Antimicrobial therapy was mainly based on the ATS/ IDSA guidelines (combined with the clinical judgment of individual primary care physicians) [16]. Appropriate antimicrobial therapy was defined as administration of at least one antimicrobial agent, to which the causative pathogen was susceptible, within 48 hours after the onset of bacteremia, with an approved route and dosage for end organ function. Antimicrobial therapy that did not meet this definition was considered as inappropriate. Monotherapy with an aminoglycoside was not considered to be appropriate therapy. The primary outcome measure was all-cause 14-day mortality following the onset of bacteremia.

\section{Microbiological Studies}

Only the first blood culture from patients with two or more positive blood cultures was included in the analysis. The presumptive identification of the isolates to the level of A. baumannii was performed with the API ID 32 GN system (bioMérieux, Marcy l'Etoile, France) or Vitek 2 system (bioMérieux, Marcy l'Etoile, France). A multiplex-PCR method was used to identify A. baumannii to the genomic species level [19]. Antimicrobial susceptibilities were determined by the agar dilution method according to the Clinical Laboratory Standards Institute (CLSI) [20]. Multidrug resistance was defined as resistance to three or more of the following classes of antimicrobial agents: anti-pseudomonal cephalosporins, anti-pseudomonal carbapenems, ampicillin/sulbactam, fluoroquinolones, and aminoglycosides [21].

\section{Molecular typing}

The clonal relationships of the clinical isolates were determined by pulsed-field gel electrophoresis (PFGE). 
PFGE of ApaI-digested genomic DNA was performed using the Bio-Rad CHEF-Mapper apparatus (Bio-Rad Laboratories, Hercules, CA, USA). DNA restriction patterns were interpreted according to the criteria of Tenover et al. [22] and cluster analysis was performed using BioNumerics version 5.0 (Applied Maths, Sint-Martens-Latem, Belgium) and the unweighted pair-group method with arithmetic averages (UPGMA). The Dice correlation coefficient was used with a tolerance of $1 \%$ in order to analyze any similarities between banding patterns. In brief, isolates showing more than three DNA fragment differences and a similarity of $<80 \%$ following dendrogram analysis were considered to represent different pulsotypes.

\section{Statistical analysis}

To assess differences, the chi-square test with Yate's correction or Fisher's exact test was used to compare the discrete variables; the Student's t-test or Mann-Whitney rank sum test was used to analyze continuous variables. Logistic regression models were used to explore independent risk factors for 14-day mortality. Univariate analyses were performed separately for each of the risk factor variables to ascertain the odds ratio (OR) and 95\% confidence interval (CI). All biologically plausible variables with a $p$ value of $\leq 0.20$ in the univariate analysis exhibited by at least $10 \%$ of the patients were considered for inclusion in the logistic regression model for the multivariate analysis. A backward selection process was utilized. Time to mortality was analyzed using Kaplan-Meier survival analysis. A $p$ value $<0.05$ was considered statistically significant. All the analyses were processed with Statistical Package for the Social Sciences (SPSS) software version 18.0 (SPSS, Chicago, IL, USA).

\section{Results}

During the study period 357 patients were found to have had at least one episode of $A$. baumannii bacteremia. We excluded 209 patients with polymicrobial bacteremia (89 patients) and those with positive blood culture attributable to another source of infection (120 patients). The final population that met the criteria for entry into the study consisted of 148 patients of A. baumannii bacteremic pneumonia. Four patients were further excluded because of incomplete medical records. Among 144 patients, 48 had NVHAP and 96 had VAP. In addition to the clinical features and radiographic findings that were compatible with diagnosis of pneumonia, 93 VAP patients had positive cultures from endotracheal aspirates, three from BAL, and all NVHAP patients from sputum specimens.

The demographic and clinical characteristics of the study patients are summarized in Table 1. Compared to VAP patients, NVHAP patients acquired their infections less frequently in an ICU, earlier after admission, received intensive care for pneumonia less frequently and received fewer procedures. Nineteen NVHAP patients (12 acquired pneumonia in an ICU and 7 outside an ICU) who received intensive care in an ICU had mechanical ventilation. Comorbidities and laboratory parameters were similar between 2 groups, except that NVHAP patients had received a fewer recent surgical procedures.

The Charlson comorbidity scores on hospital admission were similar for the two groups. Patients with NVHAP had significantly lower APACHE II scores at bacteremia onset and less often presented with bilateral lung involvement on radiological examination at the time of onset of bacteremia.

To determine the molecular epidemiology of the causative pathogens, 55 (38.2\%) of the A. baumannii isolates were randomly selected for PFGE analysis. PGFE revealed 37 different pulsotypes (A to AK, Figure 1). Among the patients with NVHAP, there were 19 isolates in 16 pulsotypes: A $(n=1), I(n=1), J(n=1), K(n=4), L(n=1), O(n=1)$, $\mathrm{T}(\mathrm{n}=1), \mathrm{U}(\mathrm{n}=1), \mathrm{V}(\mathrm{n}=1), \mathrm{W}(\mathrm{n}=1), X(\mathrm{n}=1), \mathrm{Z}(\mathrm{n}=$ $1), A B(n=1), A D(n=1), A G(n=1)$, and AK $(n=1)$. In VAP patients, there were 36 isolates in 26 pulsotypes: B ( $\mathrm{n}=1), C(\mathrm{n}=1), \mathrm{D}(\mathrm{n}=2), \mathrm{E}(\mathrm{n}=1), \mathrm{F}(\mathrm{n}=1), \mathrm{G}(\mathrm{n}=$ 2), H ( $\mathrm{n}=1), \mathrm{J}(\mathrm{n}=1), \mathrm{K}(\mathrm{n}=1), \mathrm{M}(\mathrm{n}=1), \mathrm{N}(\mathrm{n}=1), \mathrm{P}$ $(\mathrm{n}=3), \mathrm{Q}(\mathrm{n}=2), \mathrm{R}(\mathrm{n}=1), \mathrm{S}(\mathrm{n}=1), \mathrm{Y}(\mathrm{n}=3), \mathrm{Z}(\mathrm{n}=2)$, $\mathrm{AA}(\mathrm{n}=2), \mathrm{AB}(\mathrm{N}=1), \mathrm{AC}(\mathrm{n}=1), \operatorname{AE}(\mathrm{n}=1), \operatorname{AF}(\mathrm{n}=1)$, AG $(n=2), A H(n=1), A I(n=1)$, and AJ $(n=1)$.

The antimicrobial susceptibility profiles of the clinical isolates of A. baumannii are shown in Table 2. Isolates from NVHAP patients exhibited significantly lower rates of resistance to all antimicrobials tested. The initial antimicrobial agents used in both group are summarized in Table 3. The selection of antimicrobial agents was similar between both groups. Anti-pseudomonal carbapenems were the most commonly used agents in both groups, followed by anti-pseudomonal cephalosporins and penicillins. More NVHAP than VAP patients received an appropriate antimicrobial therapy, but the differences were not significant $(41.7 \%$ vs. $29.2 \%, p=0.189)$.

The 14-day mortality rate was slightly higher in NVHAP patients than those with VAP ( $43.8 \%$ vs. $31.3 \%, p=0.189)$, despite the finding that patients with NVHAP had less severe illness at the onset of bacteremia and received appropriate antimicrobial therapy more often than patients with VAP. Multivariate logistic regression analysis was performed to identify potential independent risk or protective factors for 14-day mortality. Although ICU admission did not meet the criteria for entering multivariate logistic regression analysis, it was included because of its clinical importance. Patients with VAP caused by A. baumannii had a significantly lower mortality than those with NVHAP on regression analysis (odd ratio $[\mathrm{OR}]=0.201$; 95\% confidence interval $[\mathrm{CI}]=0.075-0.538$; $p=0.001)$. Patients with higher APACHE II scores (OR = $1.164 ; 95 \% \mathrm{CI}=1.103-1.229 ; p<0.001)$, use of 
Table 1 Demographic and clinical characteristics of patients with bacteremic hospital-acquired pneumonia caused by Acinetobacter baumannii ${ }^{\mathrm{a}}$

\begin{tabular}{|c|c|c|c|}
\hline \multirow[t]{2}{*}{$\begin{array}{l}\text { Demographic or } \\
\text { characteristic }\end{array}$} & $\begin{array}{l}\text { Non-ventilator- } \\
\text { associated } \\
(n=48)\end{array}$ & $\begin{array}{c}\text { Ventilator- } \\
\text { associated } \\
(n=96)\end{array}$ & \multirow[t]{2}{*}{$\begin{array}{c}p \\
\text { Value }\end{array}$} \\
\hline & \multicolumn{2}{|c|}{$\mathrm{n}\left(\%\right.$ or $\left.\mathrm{IQR}^{\mathrm{a}}\right)$} & \\
\hline Age in year & $74(54.8-81.8)$ & $\begin{array}{c}74(59.0- \\
80.0)\end{array}$ & 0.944 \\
\hline Gender, male & $40(83.3)$ & $73(76.0)$ & 0.43 \\
\hline Acquired in ICU & $12(25.0)$ & $84(87.5)^{b}$ & $<0.001$ \\
\hline $\begin{array}{l}\text { ICU admission after } \\
\text { pneumonia }\end{array}$ & $19(39.6)^{c}$ & $86(89.6)$ & $<0.001$ \\
\hline $\begin{array}{l}\text { Days of hospitalization prior to } \\
\text { bacteremia }\end{array}$ & $14.5(3.2-3.6)$ & $\begin{array}{l}22.5(12.0- \\
40.8)\end{array}$ & 0.021 \\
\hline \multicolumn{4}{|l|}{ Comorbidity } \\
\hline Charlson comorbidity score & $3(2.0-5.0)$ & $3(2.0-5.0)$ & 0.861 \\
\hline Hypertension & $22(45.8)$ & $47(49.0)$ & 0.86 \\
\hline Coronary artery disease & $14(29.2)$ & 15 (15.6) & 0.091 \\
\hline Congestive heart failure & $6(12.5)$ & $19(19.8)$ & 0.392 \\
\hline Cerebral vascular disease & $7(14.6)$ & $27(28.1)$ & 0.111 \\
\hline COPD & $12(25.0)$ & $27(28.1)$ & 0.842 \\
\hline $\begin{array}{l}\text { Non-COPD chronic lung } \\
\text { disease }\end{array}$ & $5(10.4)$ & 15 (15.6) & 0.551 \\
\hline Alcohol & $3(6.3)$ & $13(13.5)$ & 0.302 \\
\hline Liver cirrhosis & $4(8.3)$ & $5(5.2)$ & 0.481 \\
\hline Chronic kidney disease & $15(31.3)$ & $32(33.3)$ & 0.95 \\
\hline Type 2 diabetes & $10(20.8)$ & $35(36.5)$ & 0.086 \\
\hline Collagen vascular disease & $4(8.3)$ & $7(7.3)$ & 1 \\
\hline $\begin{array}{l}\text { Usage of } \\
\text { immunosuppressants }\end{array}$ & $18(37.5)$ & $32(33.3)$ & 0.757 \\
\hline Usage of corticosteroids & $7(14.6)$ & $23(24.0)$ & 0.276 \\
\hline Neutropenia & $4(8.3)$ & $7(7.3)$ & 1 \\
\hline Malignancy & $22(45.8)$ & $28(29.2)$ & 0.073 \\
\hline $\begin{array}{l}\text { Hematologic } \\
\text { malignancy }\end{array}$ & $7(14.6)$ & $6(6.3)$ & 0.125 \\
\hline Solid malignancy & $17(35.4)$ & $23(24.0)$ & 0.211 \\
\hline Previous shock & $8(16.7)$ & $27(28.1)$ & 0.192 \\
\hline Recent surgery & $8(16.7)$ & $34(35.4)$ & 0.032 \\
\hline Trauma & $0(0)$ & $4(4.2)$ & 0.301 \\
\hline \multicolumn{4}{|l|}{ Procedures $^{d}$} \\
\hline Abdominal drain & $1(2.1)$ & $5(5.2)$ & 0.658 \\
\hline Arterial line & $5(10.4)$ & $36(37.5)$ & 0.001 \\
\hline Central venous catheter & $22(45.8)$ & $71(74.0)$ & 0.002 \\
\hline Pulmonary artery catheter & $6(12.5)$ & $20(20.8)$ & 0.319 \\
\hline Foley catheter & $28(58.3)$ & $78(81.3)$ & 0.006 \\
\hline Hemodialysis & $4(8.3)$ & $10(10.4)$ & 0.774 \\
\hline Nasogastric tube & $35(72.9)$ & $93(96.9)$ & $<0.001$ \\
\hline Thoracic drain & $0(0)$ & $7(7.3)$ & 0.095 \\
\hline
\end{tabular}

Table 1 Demographic and clinical characteristics of patients with bacteremic hospital-acquired pneumonia caused by Acinetobacter baumannii ${ }^{\text {a }}$ (Continued)

\begin{tabular}{|c|c|c|c|}
\hline Total parental nutrition & $1(2.1)$ & $8(8.3)$ & 0.273 \\
\hline Tracheostomy & 7 (14.6) & $16(16.7)$ & 0.936 \\
\hline Shock $^{d}$ & $29(60.4)$ & $58(60.4)$ & 1 \\
\hline APACHE $\|$ score $^{d}$ & $24(17.3-31.8)$ & $\begin{array}{c}27(21.3- \\
34.8)\end{array}$ & 0.015 \\
\hline \multicolumn{4}{|l|}{ Radiological features } \\
\hline $\begin{array}{l}\text { Bilateral radiologic } \\
\text { involvement }\end{array}$ & $27(56.3)$ & $81(84.4)$ & 0.001 \\
\hline Pleural effusion & $20(41.7)$ & $32(33.3)$ & 0.425 \\
\hline $\begin{array}{l}\text { Appropriate antimicrobial } \\
\text { therapy }\end{array}$ & $20(41.7)$ & $28(29.2)$ & 0.189 \\
\hline $\begin{array}{l}\text { Combination antimicrobial } \\
\text { therapy }\end{array}$ & $4(8.3)$ & $7(7.3)$ & 1 \\
\hline \multicolumn{4}{|l|}{ Outcome } \\
\hline 14-day mortality & $21(43.8)$ & $30(31.3)$ & 0.196 \\
\hline
\end{tabular}

${ }^{\mathrm{a}}$ Data are median value (interquartile range) for continuous variables and number of cases (\%) for categorical variables. IQR = interquartile range; $\mathrm{ICU}=$ intensive care unit; $\mathrm{COPD}=$ chronic obstructive pulmonary disease; APACHE = Acute Physiologic and Chronic Health Evaluation.

${ }^{\mathrm{b}}$ Other patients were cared in a respiratory care center, respiratory ward, or common ward.

Twelve acquired pneumonia in an ICU and 7 outside an ICU, all of them received mechanical ventilation after pneumonia.

${ }^{\mathrm{d}}$ At the time the blood culture was obtained.

corticosteroids $(\mathrm{OR}=5.739 ; 95 \% \mathrm{CI}=2.002-16.454 ; p=$ $0.001)$ and solid malignancies $(\mathrm{OR}=4.242 ; 95 \% \mathrm{CI}=$ 1.554-11.578; $p=0.005$ ) also had a significantly higher 14-day mortality regardless of the use of ventilators. ICU admission was not found to be a protective factor for 14-day mortality.

\section{Discussion}

In this study, we found that patients with $A$. baumannii bacteremia with NVHAP or VAP had similar demographic characteristics and Charlson comorbidity scores at hospital admission. Patients with NVHAP differed from VAP in that fewer acquired their infections in an ICU and they developed pneumonia sooner after admission. They received intensive care for pneumonia less often and had fewer invasive and surgical procedures. Patients with NVHAP also had lower APACHE II scores at the onset of bacteremia. They presented with bilateral lung involvement less often and had fewer antimicrobial resistant strains. The crude 14-day mortality rate was slightly higher in NVHAP patients than those with VAP. Following adjustment for multiple risk factors by logistic regression analysis, patients with VAP had lower 14-day mortality than those with NVHAP.

The findings on regression analysis appear to be counter-intuitive since patients with NVHAP were less ill and did not appear to require use of a ventilator. They would not be expected to be better off if treated with a 


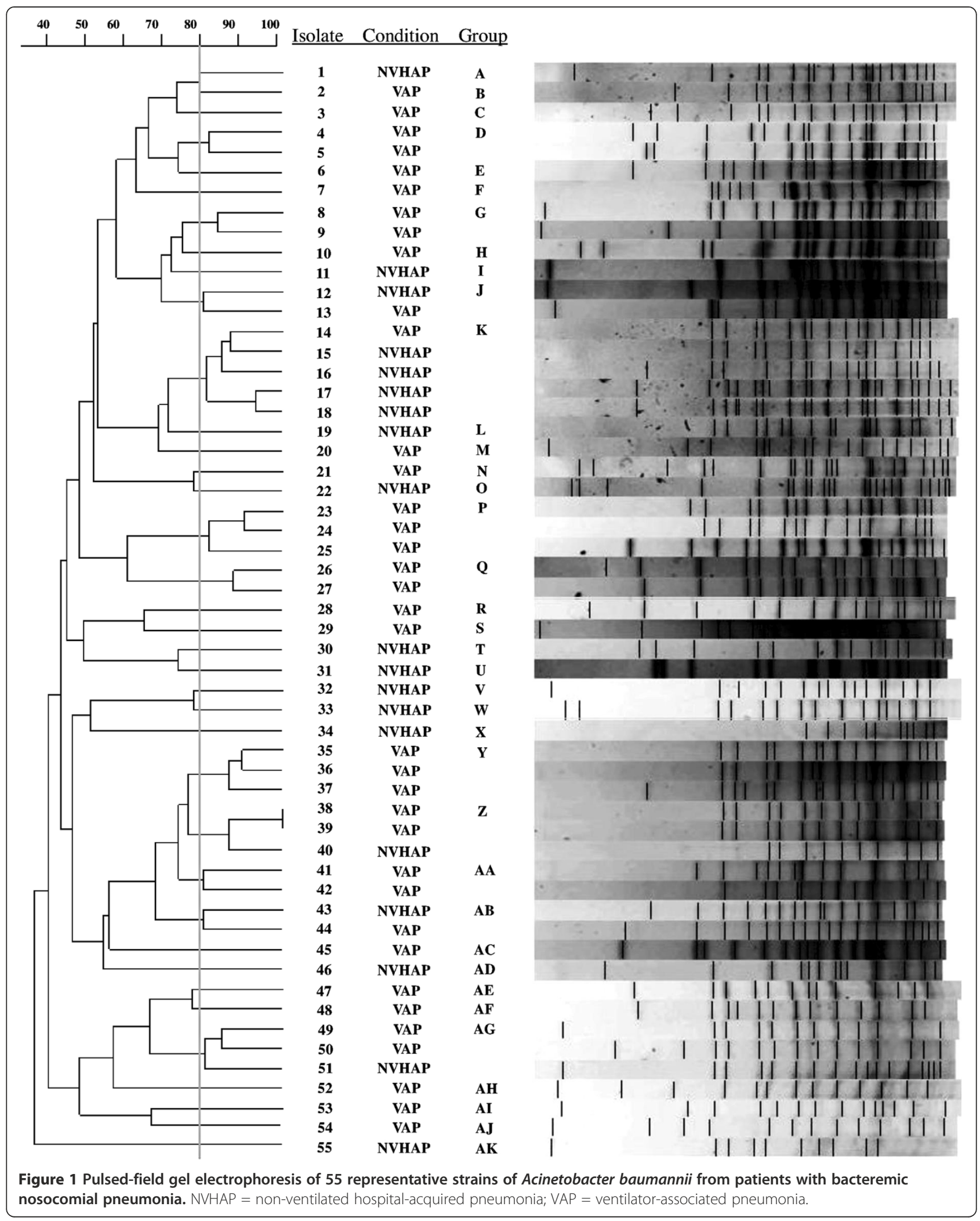


Table 2 Comparison of antimicrobial susceptibilities of blood isolates in patients with non-ventilator and ventilatorassociated hospital-acquired pneumonia

\begin{tabular}{lccc}
\hline & \multicolumn{3}{c}{ Resistance, $\mathbf{n}(\%)$} \\
Antimicrobial agent & Non-ventilator associated $(\mathbf{n}=\mathbf{4 8})$ & Ventilator-associated $(\mathbf{n}=\mathbf{9 6})$ & $<$ Value \\
\hline Amikacin & $27(56.3)$ & $86(89.6)$ & $<0.001$ \\
Gentamicin & $32(68.1)$ & $88(93.6)$ & $<0.001$ \\
Ceftazidime & $30(63.8)$ & $93(96.9)$ & $<0.001$ \\
Cefepime & $22(45.8)$ & $73(76.0)$ & 0.001 \\
Piperacillin/tazobactam & $25(52.1)$ & $77(80.2)$ & 0.001 \\
Ampicillin/sulbactam & $17(36.2)$ & $62(65.3)$ & 0.002 \\
Ciprofloxacin & $33(68.8)$ & $93(96.9)$ & $<0.001$ \\
Imipenem & $13(27.1)$ & $47(49.0)$ & 0.020 \\
Multidrug resistance ${ }^{a}$ & $31(64.6)$ & $94(97.9)$ & $<0.001$ \\
\hline
\end{tabular}

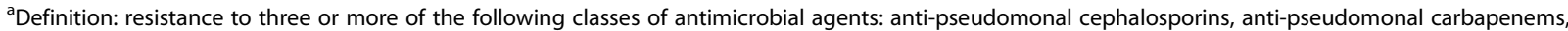
ampicillin/sulbactam, fluoroquinolones, and aminoglycosides.

ventilator, but this remains a possibility since it is difficult to assess individual clinical decisions in critically ill patients. It is reasonable to suppose that since most VAP patients were managed in ICUs, ventilator use may have been a surrogate marker for better and more intensive care. The upper airways of ventilated patients are better protected from contaminated secretions and their sputum is more readily expectorated $[23,24]$. The VAP patients might have been diagnosed and received antimicrobial therapy earlier.

Prompt use of appropriate antibiotics is essential to optimize the outcome of HAP [25-27]. A recent study showed that appropriate antimicrobial therapy reduced the mortality of bacteremic A. baumannii infections [11]. In the current study the isolates from patients with VAP were more likely than those with NVHAP to be resistant to multiple antimicrobial agents. This probably accounted for the tendency for less patients with VAP to receive appropriate therapy. Nevertheless, the frequency of antimicrobial resistance and inappropriate treatment was high in both groups. This finding is not surprising since $A$. baumannii is often resistant to the antimicrobial agents used for empirical therapy of patients with HAP. Routine respiratory surveillance cultures might be helpful to guide the selection of the most appropriate empirical therapy [28,29]. This should be particularly useful in institutions where multidrug resistant pathogens are endemic. The antimicrobial susceptibility of 1,160 strains of Acinetobacter species isolated during a 10-year nationwide survey in Taiwan was recently reported [30]. The overall susceptibility to tigecycline and colistin was $97.7 \%$ and $99.8 \%$, respectively. The susceptibility of carbapenem resistant isolates to these drugs was $98.1 \%$ and $100 \%$ respectively [30]. More prompt use of these antimicrobial agents should improve use of appropriate antimicrobial therapy. These drugs were not available at our hospital during the study period.

It is also possible that there were important differences in the pathogenicity of the invading microorganism in the

Table 3 Comparison of initial antimicrobial agents use in patients with non-ventilator and ventilator-associated hospital-acquired pneumonia

\begin{tabular}{|c|c|c|c|}
\hline \multirow[t]{2}{*}{ Main agents used } & \multicolumn{2}{|c|}{ Group, n (\%) } & \multirow{2}{*}{$\begin{array}{c}p \\
\text { Value }\end{array}$} \\
\hline & Non-ventilator associated $(n=48)$ & Ventilator-associated $(n=96)$ & \\
\hline Anti-pseudomonal penicillins ${ }^{a}$ & $5(10.4)$ & $10(10.4)$ & 1.000 \\
\hline Anti-pseudomonal cephalosporins ${ }^{b}$ & $12(25.0)$ & $18(18.8)$ & 0.514 \\
\hline Anti-pseudomonal fluoroquinolones ${ }^{c}$ & $4(8.3)$ & $4(4.2)$ & 0.441 \\
\hline Anti-pseudomonal carbapenems ${ }^{d}$ & $14(29.2)$ & $29(30.2)$ & 1.000 \\
\hline Ampicillin/sulbactam or sulbactam & $5(10.4)$ & $13(13.5)$ & 0.789 \\
\hline Non-anti-pseudomonal $\beta$-lactamase ${ }^{e}$ & $6(12.5)$ & $16(16.7)$ & 0.682 \\
\hline Miscellaneous ${ }^{f}$ & $2(4.2)$ & $6(6.3)$ & 0.719 \\
\hline
\end{tabular}

Including piperacillin, piperacillin/tazobactam, and ticarcillin/clavulanate.

bIncluding cefoperazone, ceftazidime, cefepime, and cefpirome.

'Including ciprofloxacin and levofloxacin.

${ }^{\mathrm{d}}$ Including imipenem and meropenem.

encluding penicillin, amoxicillin/clavulanate, cefazolin, cefuroxime, cefotaxime, cefmetazole, and flomoxef.

f Including teicoplanin or clindamycin plus amikacin. 
NVHAP and VAP populations. This concept is based on the higher frequency of antibiotic resistant strains among patients with VAP. It has been shown that the survival fitness and virulence of bacteria are often compromised in isolates with multidrug resistance [31,32]. PFGE analysis did not reveal a specific epidemic clone of $A$. baumannii among the two groups, but we cannot exclude the possibility that patients with VAP were infected by less invasive or less virulent clones. Another possible explanation is that patients with NVHAP may have been more susceptible to infection with $A$. baumannii than those with VAP because they developed bacteremic pneumonia without the need for intubation and mechanical respiration.

The APACHE II score at the onset of A. baumannii bacteremia has been shown to be a reliable parameter for predicting mortality [33]. In the current study we found that APACHE II score to be an independent risk factor for mortality in patients with A. baumannii HAP. In addition, solid malignancies and usage of corticosteroids were identified as independent risk factors for mortality in this study. The proportion of malignancies was higher in NVHAP patients (46\% vs. $29 \%$ ), though there was no significant difference between the two groups, but following regression analysis, solid malignancies was an independent risk factor for mortality. The higher proportion of malignancies may also play a role in the poorer outcome of NVHAP patients. The findings posed important information in risk stratification for A. baumannii HAP.

This is the first study specifically designed to compare the outcome of NVHAP and VAP bacteremic A. baumannii pneumonia. The strengths of this study are the relatively large number of patients managed in a major tertiary care teaching hospital using well defined criteria for underlying diseases, examination of clonal relationships and a clear 14-day end-point. The limitations include the difficulty to differentiate colonization from infection in sputum cultures of patients with HAP. To improve the specificity of the quantitative sputum cultures we excluded patients with A. baumannii bacteremia that could not be attributed to pneumonia. Concomitant catheter related bacteremia or secondary catheter infection could not be completely excluded because central venous catheter cultures were unavailable. Because of the retrospective design we were unable to control the selection of the antimicrobial agents prescribed by the hospital staff. However, there were no significant differences between the VAP and NVHAP in respect to the choice of drugs and variety of antimicrobial regimens that might have affected the clinical outcomes.

\section{Conclusions}

Patients with bacteremic NVHAP and VAP caused by A. baumannii had similar crude mortality rates, but on logistic regression analysis those receiving ventilator assistance had a significantly lower mortality. This may have been due to better airway protection, more intensive monitoring with earlier diagnosis and treatment in patients with VAP, greater innate susceptibility to infection in those with NVHAP and differences in the virulence of $A$. baumannii.

\section{Abbreviations}

A. baumannii: Acinetobacter baumannii; APACHE II: Acute Physiology And Chronic Health Evaluation II; ATS/IDSA: American Thoracic Society/Infectious Diseases Society of America; BAL: Broncho-alveolar lavage; Cl: Confidence interval; CLSI: Clinical Laboratory Standards Institute; COPD: Chronic obstructive pulmonary disease; DNA: Deoxyribonucleic acid; HAP: Hospital-acquired pneumonia; ICU: Intensive care unit; NVHAP: Non-ventilated HAP; OmpA: Outer membrane protein $A ;$ OR: Odds ratio; PCR: Polymerase chain reaction; PFGE: Pulsed-field gel electrophoresis; SPSS: Statistical Package for the Social Sciences; TSGH: Tri-Service General Hospital; T-VGH: Taipei Veterans General Hospital; UPGMA: Unweighted pair-group method with arithmetic averages; VAP: Ventilator-associated pneumonia.

\section{Competing interests}

Te-Li Chen is a medical advisor of TTY Biopharm. Other authors declare that they have no competing interests.

\section{Authors' contributions}

YSY, YTL and TLC participated in the study design, analysis of data, and writing of the manuscript. JRS and SCK participated in data collection. TWH and CHY participated in analysis of data. JCL, CPF and FYC revised the manuscript with important intellectual contribution. All authors read and approved the final manuscript.

\section{Acknowledgements}

The authors wish to express their appreciation to Calvin M. Kunin for his critical review of this manuscript.

This study was supported by grants from the Tri-Service General Hospital (TSGH-C100-103, TSGH-C102-113, MAB101-03 and MAB102-13),the Taipei Veterans General Hospital (V101E4-003, V101A-017, and V101C-021), the National Science Council (101-2314-B-010-027-MY3), and the National Health Research Institute (IV-101-PP-12).

\section{Author details}

'Division of Infectious Diseases and Tropical Medicine, Department of Internal Medicine, Tri-Service General Hospital, National Defense Medical Center, Taipei, Taiwan. ${ }^{2}$ Institute of Clinical Medicine, School of Medicine, National Yang-Ming University, Taipei, Taiwan. ${ }^{3}$ Emergency Department, Taipei Veterans General Hospital, Taipei, Taiwan. ${ }^{4}$ Division of Thoracic Surgery, Department of Surgery, Tri-Service General Hospital, National Defense Medical Center, Taipei, Taiwan. ${ }^{5}$ Clinical Microbiology Laboratory Division of Clinical Pathology, Tri-Service General Hospital, National Defense Medical Center, Taipei, Taiwan. ${ }^{6}$ National Institute of Infectious Diseases and Vaccinology, National Health Research Institutes, Miaoli County, Taiwan.

${ }^{7}$ Department of Health, Centers for Disease Control, Taipei, Taiwan.

Received: 20 November 2012 Accepted: 12 March 2013

Published: 19 March 2013

\section{References}

1. Luna CM, Aruj PK: Nosocomial Acinetobacter pneumonia. Respirology 2007, 12(6):787-791.

2. Cisneros JM, Reyes MJ, Pachon J, Becerril B, Caballero FJ, Garcia-Garmendia JL, Ortiz C, Cobacho AR: Bacteremia due to Acinetobacter baumannii: epidemiology, clinical findings, and prognostic features. Clin Infect Dis 1996, 22(6):1026-1032.

3. Garcia-Garmendia JL, Ortiz-Leyba C, Garnacho-Montero J, Jimenez-Jimenez FJ, Perez-Paredes C, Barrero-Almodovar AE, Gili-Miner M: Risk factors for Acinetobacter baumannii nosocomial bacteremia in critically ill patients: a cohort study. Clin Infect Dis 2001, 33(7):939-946.

4. Levin AS, Levy CE, Manrique AE, Medeiros EA, Costa SF: Severe nosocomial infections with imipenem-resistant Acinetobacter baumannii treated with ampicillin/sulbactam. Int J Antimicrob Agents 2003, 21(1):58-62. 
5. Seifert H, Strate A, Pulverer G: Nosocomial bacteremia due to Acinetobacter baumannii. Clinical features, epidemiology, and predictors of mortality. Medicine (Baltimore) 1995, 74(6):340-349.

6. Joly-Guillou ML: Clinical impact and pathogenicity of Acinetobacter. Clin Microbiol Infect 2005, 11(11):868-873.

7. Lee YC, Huang YT, Tan CK, Kuo YW, Liao CH, Lee PI, Hsueh PR: Acinetobacter baumannii and Acinetobacter genospecies 13 TU and 3 bacteraemia: comparison of clinical features, prognostic factors and outcomes. J Antimicrob Chemother 2011, 66(8):1839-1846.

8. Gordon NC, Wareham DW: Multidrug-resistant Acinetobacter baumannii: mechanisms of virulence and resistance. Int J Antimicrob Agents 2010, 35(3):219-226.

9. Cerqueira GM, Peleg AY: Insights into Acinetobacter baumannii pathogenicity. IUBMB life 2011, 63(12):1055-1060.

10. Chuang YC, Sheng WH, Li SY, Lin YC, Wang JT, Chen YC, Chang SC: Influence of genospecies of Acinetobacter baumannii complex on clinical outcomes of patients with Acinetobacter bacteremia. Clin Infect Dis 2011, 52(3):352-360.

11. Lee YT, Kuo SC, Yang SP, Lin YT, Tseng FC, Chen TL, Fung CP: Impact of appropriate antimicrobial therapy on mortality associated with Acinetobacter baumannii bacteremia: relation to severity of infection. Clin Infect Dis 2012, 55(2):209-215.

12. Abrahamian FM, Deblieux PM, Emerman CL, Kollef MH, Kupersmith $\mathrm{E}_{\text {, }}$ Leeper KV Jr, Paterson DL, Shorr AF: Health care-associated pneumonia: identification and initial management in the ED. Am J Emerg Med 2008, 26(6 Suppl):1-11

13. Chung DR, Song JH, Kim SH, Thamlikitkul V, Huang SG, Wang H, So TM, Yasin RM, Hsueh PR, Carlos CC, et al: High prevalence of multidrugresistant nonfermenters in hospital-acquired pneumonia in Asia. Am J Respir Crit Care Med 2011, 184(12):1409-1417.

14. Ewig S, Bauer $T$, Torres A: The pulmonary physician in critical care * 4 : Nosocomial pneumonia. Thorax 2002, 57(4):366-371.

15. Magret M, Lisboa T, Martin-Loeches I, Manez R, Nauwynck M, Wrigge H, Cardellino S, Diaz E, Koulenti D, Rello J: Bacteremia is an independent risk factor for mortality in nosocomial pneumonia: a prospective and observational multicenter study. Crit Care 2011, 15(1):R62.

16. : Guidelines for the management of adults with hospital-acquired, ventilator-associated, and healthcare-associated pneumonia. Am J Respir Crit Care Med 2005, 171(4):388-416.

17. Charlson ME, Pompei P, Ales KL, Mackenzie CR: A new method of classifying prognostic comorbidity in longitudinal studies: development and validation. J Chronic Dis 1987, 40(5):373-383.

18. Knaus WA, Draper EA, Wagner DP, Zimmerman JE: APACHE II: a severity of disease classification system. Crit Care Med 1985, 13(10):818-829.

19. Chen $T L$, Siu LK, Wu RC, Shaio MF, Huang LY, Fung CP, Lee CM, Cho WL: Comparison of one-tube multiplex PCR, automated ribotyping and intergenic spacer (ITS) sequencing for rapid identification of Acinetobacter baumannii. Clin Microbiol Infect 2007, 13(8):801-806.

20. Clinical and Laboratory Standards Institute (CLSI): Performance standards for antimicrobial susceptibility testing: Twenty-first informational supplement. CLSI, Wayne, PA: CLSI document M100-S21; 2011.

21. Peleg AY, Seifert $H$, Paterson DL: Acinetobacter baumannii: emergence of a successful pathogen. Clin Microbiol Rev 2008, 21(3):538-582.

22. Tenover FC, Arbeit RD, Goering RV, Mickelsen PA, Murray BE, Persing DH, Swaminathan B: Interpreting chromosomal DNA restriction patterns produced by pulsed-field gel electrophoresis: criteria for bacterial strain typing. J Clin Microbiol 1995, 33(9):2233-2239.

23. Esperatti M, Ferrer M, Theessen A, Liapikou A, Valencia M, Saucedo LM, Zavala E, Welte T, Torres A: Nosocomial pneumonia in the intensive care unit acquired by mechanically ventilated versus nonventilated patients. Am J Respir Crit Care Med 2010, 182(12):1533-1539.

24. Sopena N, Sabria M, Neunos Study G: Multicenter study of hospitalacquired pneumonia in non-ICU patients. Chest 2005, 127(1):213-219.

25. Kollef MH, Sherman G, Ward S, Fraser VJ: Inadequate antimicrobial treatment of infections: a risk factor for hospital mortality among critically ill patients. Chest 1999, 115(2):462-474.

26. Luna CM, Vujacich P, Niederman MS, Vay C, Gherardi C, Matera J, Jolly EC: Impact of BAL data on the therapy and outcome of ventilator-associated pneumonia. Chest 1997, 111(3):676-685.

27. Lynch JP 3rd: Hospital-acquired pneumonia: risk factors, microbiology, and treatment. Chest 2001, 119(2 Suppl):373S-384S.
28. Brusselaers N, Labeau S, Vogelaers D, Blot S: Value of lower respiratory tract surveillance cultures to predict bacterial pathogens in ventilatorassociated pneumonia: systematic review and diagnostic test accuracy meta-analysis. Intensive Care Med 2012.

29. Brusselaers N, Logie D, Vogelaers D, Monstrey S, Blot S: Burns, inhalation injury and ventilator-associated pneumonia: value of routine surveillance cultures. Burns: J Int Soc for Burn Injuries 2012, 38(3):364-370.

30. Kuo SC, Chang SC, Wang HY, Lai JF, Chen PC, Shiau YR, Huang IW, Lauderdale TL, Hospitals T: Emergence of extensively drug-resistant Acinetobacter baumannii complex over 10 years: nationwide data from the Taiwan Surveillance of Antimicrobial Resistance (TSAR) program. BMC Infect Dis 2012, 12:200.

31. Andersson DI, Hughes D: Antibiotic resistance and its cost: is it possible to reverse resistance? Nat Rev Microbio/ 2010, 8(4):260-271.

32. Kang YS, Park W: Trade-off between antibiotic resistance and biological fitness in Acinetobacter sp. strain DR1. Environmental Microbiol 2010, 12(5):1304-1318.

33. Chen SJ, Chao TF, Chiang MC, Kuo SC, Chen LY, Yin T, Chen TL, Fung CP: Prediction of patient outcome from Acinetobacter baumannii bacteremia with Sequential Organ Failure Assessment (SOFA) and Acute Physiology and Chronic Health Evaluation (APACHE) II scores. Intern Med 2011, 50(8):871-877.

\section{doi:10.1186/1471-2334-13-142}

Cite this article as: Yang et al:: Acinetobacter baumannii nosocomial pneumonia: is the outcome more favorable in non-ventilated than ventilated patients?. BMC Infectious Diseases 2013 13:142.

\section{Submit your next manuscript to BioMed Central and take full advantage of:}

- Convenient online submission

- Thorough peer review

- No space constraints or color figure charges

- Immediate publication on acceptance

- Inclusion in PubMed, CAS, Scopus and Google Scholar

- Research which is freely available for redistribution 